Noname manuscript No.

(will be inserted by the editor)

\title{
More results on Schur complements in Euclidean Jordan algebras
}

\author{
R. Sznajder · M.S. Gowda • M.M. Moldovan
}

December 31, 2009

\begin{abstract}
In a recent article [8], Gowda and Sznajder studied the concept of Schur complement in Euclidean Jordan algebras and described Schur determinantal and Haynsworth inertia formulas. In this article, we establish some more results on the Schur complement. Specifically, we prove, in the setting of Euclidean Jordan algebras, an analogue of the Crabtree-Haynsworth quotient formula and show that any Schur complement of a strictly diagonally dominant element is strictly diagonally dominant. We also introduce the concept of Schur product of a real symmetric matrix and an element of a Euclidean Jordan algebra when its Peirce decomposition with respect to a Jordan frame is given. An Oppenheim type inequality is proved in this setting.
\end{abstract}

Keywords Schur complements · Euclidean Jordan algebra · Crabtree-Haynsworth quotient formula . Carlson-Markham strict diagonal dominance theorem · Schur product · Oppenheim inequality

Roman Sznajder

Department of Mathematics

Bowie State University

Bowie, Maryland 20715, USA

E-mail: rsznajder@bowiestate.edu

M. Seetharama Gowda

Department of Mathematics and Statistics

University of Maryland, Baltimore County

Baltimore, Maryland 21250, USA

E-mail: gowda@math.umbc.edu

Melania M. Moldovan

Department of Mathematics

Technical University of Cluj-Napoca

Cluj-Napoca, Cluj 400020, ROMANIA

E-mail: melania.moldovan@math.utcluj.ro 


\section{Introduction}

Consider a complex matrix $M$ given in the block form

$$
M=\left[\begin{array}{ll}
A & B \\
C & D
\end{array}\right]
$$

with $A$ invertible. Then the Schur complement of $A$ in $M$ is given by

$$
M / A=D-C A^{-1} B .
$$

The Schur complement enjoys numerous properties such as the Schur determinantal formula, the Haynsworth inertia formula, the Guttman rank formula, etc., and appears in various applications [3], [18], [22]. The above definition continues to be valid for quaternion matrices, but does not make sense for octonion matrices for lack of associative property. In addition, lack of a nontrivial multiplicative determinant for matrices over quaternions and octonions [2] prevents one from a meaningful study of Schur complements of matrices over such numbers. However, the concept of Schur complement can be studied for square Hermitian matrices (of suitable size) over quaternions and octonions, and more generally over Euclidean Jordan algebras.

Consider a Euclidean Jordan algebra $\mathcal{J}$ (which is a finite dimensional real inner product space with a compatible Jordan product), an idempotent $c \in \mathcal{J}$ (that is, $c^{2}=c$ ), and the corresponding Peirce decomposition

$$
\mathcal{J}=\mathcal{J}(c, 1) \oplus \mathcal{J}\left(c, \frac{1}{2}\right) \oplus \mathcal{J}(c, 0)
$$

where $\mathcal{J}(c, \gamma)=\{x \in \mathcal{J}: x \circ c=\gamma x\}$ and $\gamma \in\left\{0, \frac{1}{2}, 1\right\}$. For any $x \in \mathcal{J}$, let

$$
x=u+v+w,
$$

where $u \in \mathcal{J}(c, 1), v \in \mathcal{J}\left(c, \frac{1}{2}\right)$, and $w \in \mathcal{J}(c, 0)$. When $u$ is invertible in the Euclidean Jordan (sub)algebra $\mathcal{J}(c, 1)$, let $u_{*}^{-1}$ denote the inverse of $u$ in $\mathcal{J}(c, 1)$. In this case, the Schur complement of $u$ in $x$ is defined by

$$
x / u:=w-P_{v}\left(u_{*}^{-1}\right),
$$

where, for any element $a \in \mathcal{J}$, the quadratic representation $P_{a}$ is defined on $\mathcal{J}$ by

$$
P_{a}(z)=2 a \circ(a \circ z)-(a \circ a) \circ z \quad(z \in \mathcal{J}) .
$$

This concept was introduced in [16], see also [15], where it was shown that $x / u \in \mathcal{J}(c, 0)$ and

$$
\operatorname{det}(x)=\operatorname{det}(u) \operatorname{det}(x / u) .
$$

(Here, the determinants $\operatorname{det}(x), \operatorname{det}(u)$, and $\operatorname{det}(x / u)$ are taken in the algebras $\mathcal{J}$, $\mathcal{J}(c, 1)$, and $\mathcal{J}(c, 0)$, respectively.) Based on this, Gowda and Sznajder [8] showed that

(i) $\operatorname{In}(x)=\operatorname{In}(u)+\operatorname{In}(x / u)$,

(ii) $x>0(\geq 0)$ in $\mathcal{J}$ if and only if $u>0(\geq 0)$ in $\mathcal{J}(c, 1)$ and $x / u>0(\geq 0)$ in $\mathcal{J}(c, 0)$, and

(iii) $\operatorname{rank}(x)=\operatorname{rank}(u)+\operatorname{rank}(x / u)$, 
where $\operatorname{In}(x)$ and $\operatorname{rank}(x)$ denote the inertia and rank of an element $x$ respectively, and $x \geq 0$ means that $x$ belongs to the symmetric cone in $\mathcal{J}$.

With appropriate identification, the above results reduce to the familiar results of matrix theory in the algebras $\operatorname{Herm}\left(\mathcal{R}^{n \times n}\right)$ and $\operatorname{Herm}\left(\mathcal{C}^{n \times n}\right)$ of all $n \times n$ Hermitian matrices over real numbers and complex numbers. The novelty of the results $(i)-($ iii $)$ lies in the fact that they continue to hold in $\operatorname{Herm}\left(\mathcal{H}^{n \times n}\right)$ (the algebra of all $n \times n$ Hermitian matrices over quaternions), $\operatorname{Herm}\left(\mathcal{O}^{3 \times 3}\right)$ (the algebra of all $3 \times 3$ Hermitian matrices over octonions), and the Jordan spin algebra $\mathcal{L}^{n}$.

The objective of this present paper is to describe some more results on the Schur complement in the setting of Euclidean Jordan algebras. The motivation for our work comes, once again, from classical results.

Consider a complex matrix $M$ with an invertible principal submatrix $U$. Let $A$ be an invertible principal submatrix of $U$. Then the well-known Crabtree-Haynsworth quotient formula [4] says that $U / A$ is a nonsingular principal submatrix of $M / A$ and

$$
(M / A) /(U / A)=M / U
$$

The proof of this result, see e.g., [22], Theorem 1.4, continues to hold for quaternion matrices. Our first result deals with an analogue of these in Euclidean Jordan algebras:

Theorem 1 Let $c$ and $d$ be two idempotents in a Euclidean Jordan algebra $\mathcal{J}$ with $d \leq c$. For any $x \in \mathcal{J}$, suppose that $u:=P_{c}(x)$ is invertible in $\mathcal{J}(c, 1)$ and $a:=P_{d}(u)$ is invertible in $\mathcal{J}(d, 1)$. Then $u / a=P_{c-d}(x / u)$ is invertible in $\mathcal{J}(c-d, 1)$ and

$$
(x / a) /(u / a)=x / u
$$

To describe our second result, consider a complex matrix $M=\left[m_{i j}\right]$, which is strictly diagonally dominant. This means that $\left|m_{i i}\right|>\sum_{j \neq i}\left|m_{i j}\right|$ for all $i$. The Carlson-Markham Theorem [1] says that if $U$ is any principal submatrix of $M$, then $U$ and $M / U$ are strictly diagonally dominant. Now consider a general Euclidean Jordan algebra $\mathcal{J}$. We say that $x$ in $\mathcal{J}$ is strictly diagonally dominant [17] with respect to a Jordan frame $\left\{e_{1}, \ldots, e_{r}\right\}$, if the corresponding Peirce decomposition

$$
x=\sum_{i=1}^{r} x_{i} e_{i}+\sum_{1 \leq i<j \leq r} x_{i j}
$$

satisfies the inequalities

$$
\left|x_{k}\right|>\frac{1}{\sqrt{2}|| e_{k} \|}\left(\sum_{i=1}^{k-1}\left\|x_{i k}\right\|+\sum_{j=k+1}^{r}\left\|x_{k j}\right\|\right) \forall k=1,2, \ldots, r .
$$

In this paper, we prove the following generalization:

Theorem 2 Given a Jordan frame $\left\{e_{1}, \ldots, e_{r}\right\}$ in $\mathcal{J}$ and $1 \leq k \leq r$, let $c:=$ $e_{1}+e_{2}+\cdots+e_{k}$ and $x=u+v+w$ as in (2). If $x$ is strictly diagonally dominant with respect to $\left\{e_{1}, \ldots, e_{r}\right\}$, then $u \in \mathcal{J}(c, 1)$ is strictly diagonally dominant with respect to $\left\{e_{1}, \ldots, e_{k}\right\}$ and $x / u \in \mathcal{J}(c, 0)$ is strictly diagonally dominant with respect to $\left\{e_{k+1}, \ldots, e_{r}\right\}$. 
Our next set of results deals with analogues of the Schur product (also known as Hadamard product), Schur's theorem, and Oppenheim's inequality in Euclidean Jordan algebras. Given two real or complex matrices $A=\left[a_{i j}\right]$ and $X=\left[x_{i j}\right]$, the Schur product is given by $A \circ X=\left[a_{i j} x_{i j}\right]$. The well-known Schur's theorem [13] says that if $A$ and $X$ are both Hermitian and positive semidefinite, then so is $A \circ X$. In this setting, the Oppenheim inequality says that,

$$
\left(a_{11} a_{22} \cdots a_{r r}\right) \operatorname{det}(X) \leq \operatorname{det}(A \circ X) \leq\left(a_{11} a_{22} \cdots a_{r r}\right)\left(x_{11} x_{22} \cdots x_{r r}\right) .
$$

Now let $A=\left[a_{i j}\right]$ be an $r \times r$ real symmetric matrix and $x=\sum_{i \leq j} x_{i j}$ be the Peirce decomposition of an $x \in \mathcal{J}$ with respect to a Jordan frame $\left\{e_{1}, \ldots, e_{r}\right\}$. Then we define the Schur product of $A$ and $x$ by

$$
A \bullet x:=\sum_{i \leq j} a_{i j} x_{i j} .
$$

We show that if $A$ is positive semidefinite and $x \geq 0$ in $\mathcal{J}$, then $A \bullet x \geq 0$ in $\mathcal{J}$ and satisfies an Oppenheim type inequality.

Here is an outline of our paper. In Section 2, we cover some basic material related to Euclidean Jordan algebras. Section 3 deals with an analogue of the CrabtreeHaynsworth quotient formula. In Section 4, we prove results on strict diagonal dominance and finally in Section 5, present results related to the Schur product.

\section{Preliminaries}

Throughout this paper, we let $(\mathcal{J},\langle\cdot, \cdot\rangle, \circ)$ denote a Euclidean Jordan algebra of rank $r$ [6], [9], [20]. The symmetric cone of $\mathcal{J}$ is the cone of squares $K:=\left\{x^{2}: x \in \mathcal{J}\right\}$. We use the notation $x \geq 0 \quad(x>0)$ when $x \in K$ (respectively, $x \in$ interior $(K))$. Let $\mathcal{F}$ denote the set of all real numbers/complex numbers/quaternions/octonions. We write $\operatorname{Herm}\left(\mathcal{F}^{n \times n}\right)$ for the space of all $n \times n$ Hermitian matrices with entries from $\mathcal{F}$. With $n=3$ for octonions and no restriction for others, $\operatorname{Herm}\left(\mathcal{F}^{n \times n}\right)$ becomes a Euclidean Jordan algebra with the Jordan product given by $X \circ Y=\frac{X Y+Y X}{2}$ and inner product given by $\langle X, Y\rangle=\operatorname{Re} \operatorname{tr}(X Y)$. As is well known, any Euclidean Jordan algebra is a product of simple Euclidean Jordan algebras and every simple algebra is isomorphic to the Jordan spin algebra $\mathcal{L}^{n}$ or to the algebra of all $n \times n$ real/complex/quaternion Hermitian matrices or to the algebra of all $3 \times 3$ octonion Hermitian matrices.

Any element $x \in \mathcal{J}$ will have a spectral decomposition

$$
x=\lambda_{1} e_{1}+\lambda_{2} e_{2}+\cdots+\lambda_{r} e_{r}
$$

where $\left\{e_{1}, e_{2}, \ldots, e_{r}\right\}$ is a Jordan frame in $\mathcal{J}$ and real numbers $\lambda_{1}, \lambda_{2}, \ldots, \lambda_{r}$ are the (spectral) eigenvalues of $x$. Then the determinant and trace are defined by

$$
\operatorname{det}(x):=\lambda_{1} \lambda_{2} \cdots \lambda_{r} \quad \text { and } \operatorname{tr}(x):=\lambda_{1}+\lambda_{2}+\cdots+\lambda_{r} .
$$

We remark that for Hermitian matrices over real numbers/complex numbers/quaternions, these spectral eigenvalues coincide with the real right eigenvalues; they can be different in the case of octonions [5], [17]. 
It is well known that in $\mathcal{J},\langle x, y\rangle_{t r}:=\operatorname{tr}(x \circ y)$ defines another compatible inner product (which we call as the trace inner product). In this inner product, the norm of any primitive idempotent is one.

For any $a \in \mathcal{J}$, we define the Lyapunov transformation $L_{a}$ by

$$
L_{a}(x)=a \circ x
$$

Two elements $a$ and $b$ in $\mathcal{J}$ are said to operator commute if $L_{a} L_{b}=L_{b} L_{a}$ (which happens if and only if $a$ and $b$ have their spectral decompositions with respect to a common Jordan frame).

Let $\left\{e_{1}, e_{2}, \ldots, e_{r}\right\}$ be a fixed Jordan frame in $\mathcal{J}$. For $i, j \in\{1,2, \ldots, r\}$, consider the eigenspaces

$$
\mathcal{J}_{i i}:=\left\{x \in \mathcal{J}: x \circ e_{i}=x\right\}=\mathcal{R} e_{i}
$$

and when $i \neq j$,

$$
\mathcal{J}_{i j}:=\left\{x \in \mathcal{J}: x \circ e_{i}=\frac{1}{2} x=x \circ e_{j}\right\}
$$

Then the space $\mathcal{J}$ is the orthogonal direct sum of spaces $\mathcal{J}_{i j}(i \leq j)$. Thus, given any Jordan frame $\left\{e_{1}, e_{2}, \ldots, e_{r}\right\}$, we can write any element $x \in \mathcal{J}$ as

$$
x=\sum_{1 \leq i \leq j \leq r} x_{i j}=\sum_{i=1}^{r} x_{i} e_{i}+\sum_{1 \leq i<j \leq r} x_{i j},
$$

where $x_{i} \in \mathcal{R}$ and $x_{i j} \in \mathcal{J}_{i j}$. We refer to this as the Peirce decomposition of $x$ with respect to the given Jordan frame. When $c=e_{1}+e_{2}+\cdots+e_{k}$ for $k \leq r$, the decompositions (2) and (7) are related by

$$
x=\sum_{1 \leq i \leq j \leq r} x_{i j}=u+v+w, \quad u=\sum_{1 \leq i \leq j \leq k} x_{i j}, \text { and } w=\sum_{k+1 \leq i \leq j \leq r} x_{i j} .
$$

In this case, the quadratic representation $P_{c}$ is nothing but the projection onto $\mathcal{J}(c, 1)$ ([6], Theorem IV.2.1). In particular, $P_{c}(x)=u$.

In the process of proving Theorems 1 and 2, we will need to reduce or replace a general Euclidean Jordan algebra by a simple algebra, any Jordan frame by a specific Jordan frame, and the inner product by the trace inner product. We justify this reduction process by means of the following remarks [6].

Any Euclidean Jordan algebra is a product of simple algebras. In this setting, the Jordan product is given componentwise and the inner product is the sum of inner products on each of the simple algebras. Also, all the components of an idempotent in the product algebra are idempotents; for a primitive idempotent, all these components are zero except for one component which is a primitive idempotent (in the constituent component algebra). As a consequence, the Peirce spaces $\mathcal{J}_{i j}$ (defined above) corresponding to primitive idempotents $e_{i}$ and $e_{j}$ coming from different component algebras will be zero. In addition, Schur complements and Peirce decompositions - whether of the form (1) or (7) - can be computed/described by working with the components. In each simple algebra, the inner product is a positive multiple of the trace inner product ([6], Prop. III.4.1) and any Jordan frame can be mapped onto any other by an algebra automorphism (which preserves the Jordan product as well as the inner product), see [6], Theorem IV.2.5. 
Lemma 1 In $\mathcal{J}$, consider two idempotents $c$ and $d$ with $d \leq c$. Then

(i) $d \in \mathcal{J}(c, 1)$. In addition, $d$ and $c$ operator commute.

(ii) $\mathcal{J}(d, 1) \subseteq \mathcal{J}(c, 1)$ and $\mathcal{J}(c, 0) \subseteq \mathcal{J}(d, 0)$.

(iii) $\mathcal{J}(c, 1) \cap \mathcal{J}(d, 0)=\mathcal{J}(c-d, 1)$.

Proof. (i) As $d \leq c$, we write $c=d+f$ with $f \geq 0$. Since $\mathcal{J}(c, 1)$ is a subalgebra and $\mathcal{J}(c, 1) \cap K$ is a face of $K$ (see [7], Theorem 3.1) with $c \in \mathcal{J}(c, 1)$, we must have $d, f \in \mathcal{J}(c, 1)$. As $c$ is the unit element in $\mathcal{J}(c, 1), c$ and $d$ operator commute.

(ii) Let $x \in \mathcal{J}(d, 1) \cap K$. Then $x \circ d=x$ implies that $x \circ(e-d)=0$. This gives $0=\langle x, e-d\rangle=\langle x,(e-c)+f\rangle=0$. As $x \geq 0$ and $c \leq e$, this leads to $\langle x, e-c\rangle=0$ and to $x \circ(e-c)=0$, see [9], Prop. 6. Thus $x \circ c=x$. The inclusion $\mathcal{J}(d, 1) \subseteq \mathcal{J}(c, 1)$ follows from the observation that in the algebra $\mathcal{J}(d, 1)$, any element can be written as the difference of two elements in $\mathcal{J}(d, 1) \cap K$. Finally, $d \leq c$ implies that $e-c \leq e-d$ and $\mathcal{J}(c, 0)=\mathcal{J}(e-c, 1) \subseteq \mathcal{J}(e-d, 1)=\mathcal{J}(d, 0)$.

(iii) The proof of (ii) works for $f=c-d \leq c$ in place of $d$. Thus, $\mathcal{J}(f, 1) \subseteq \mathcal{J}(c, 1)$. This implies that $\mathcal{J}(f, 1) \subseteq \mathcal{J}(d, 0)$. Hence we have one inclusion in the equality of sets in $($ iii $)$. The other inclusion is obvious.

Remarks. Given $d \leq c$ as in the above lemma, consider the decompositions $\mathcal{J}=$ $\mathcal{J}(c, 1)+\mathcal{J}\left(c, \frac{1}{2}\right)+\mathcal{J}(c, 0)$ and $\mathcal{J}(c, 1)=: G=G(d, 1)+G\left(d, \frac{1}{2}\right)+G(d, 0)$. The above lemma implies that

$$
G(d, 1)=\mathcal{J}(d, 1) \quad \text { and } \quad G(d, 0)+\mathcal{J}(c, 0) \subseteq \mathcal{J}(d, 0) .
$$

We claim that $G(d, 0) \perp \mathcal{J}(c, 0)$. To see this, let $0 \leq u \in G(d, 0)$ and $0 \leq v \in \mathcal{J}(c, 0)$. Then $v \circ c=0$ implies that $\langle v, d+f\rangle=0$. This yields, $\langle v, f\rangle=0$ and $f \circ v=0$. Now $u \circ d=0$ implies $u \circ f=u$ and $\langle u, v\rangle=\langle f \circ u, v\rangle=\langle u, f \circ v\rangle=0$.

\section{The Crabtree-Haynsworth quotient formula in Euclidean Jordan algebras}

The goal of this section is to prove Theorem 1 in a general Euclidean Jordan algebra. In view of the reduction process described in Section 2, it is enough to prove the result in each of the four matrix algebras and in $\mathcal{L}^{n}$. The result is vacuously true in $\mathcal{L}^{n}$, as the rank of $\mathcal{L}^{n}$ is two. Let $\mathcal{J}$ be any one of the matrix algebras $\operatorname{Herm}\left(\mathcal{R}^{n \times n}\right)$, $\operatorname{Herm}\left(\mathcal{C}^{n \times n}\right), \operatorname{Herm}\left(\mathcal{H}^{n \times n}\right)$, and $\operatorname{Herm}\left(\mathcal{O}^{3 \times 3}\right)$. Consider the nontrivial case of idempotents $d$ and $c$ with $d \leq c, d \neq c \neq e$. As shown in Lemma $1, d$ and $c$ commute. In $\mathcal{J}$, there exists a Jordan frame $\left\{e_{1}, e_{2}, \ldots, e_{r}\right\}$ such that

$$
d=e_{1}+e_{2}+\cdots+e_{l} \text { and } c=e_{1}+e_{2}+\cdots+e_{l}+e_{l+1}+\cdots+e_{k},
$$

where $1 \leq l<k<r$. In these matrix (simple) algebras, we can transform, by means of an algebra automorphism, the Jordan frame $\left\{e_{1}, e_{2}, \ldots, e_{r}\right\}$ into the canonical Jordan frame $\left\{E_{1}, E_{2}, \ldots, E_{r}\right\}$, where $E_{i}$ denotes a matrix with one in the $(i, i)$ slot and zeros elsewhere. (From now on, we use capital letters to denote matrices.) Let $C:=E_{1}+$ $E_{2}+\cdots+E_{k}$ and $D:=E_{1}+E_{2}+\cdots+E_{l}$. In the algebras $\operatorname{Herm}\left(\mathcal{R}^{n \times n}\right), \operatorname{Herm}\left(\mathcal{C}^{n \times n}\right)$, and $\operatorname{Herm}\left(\mathcal{H}^{n \times n}\right)$, any quadratic representation is given by $P_{X}(Y)=X Y X$. Hence for given $C$ and $D$ as above, with appropriate identification, our Schur complement corresponds to the classical Schur complement. Now, in the algebras $\operatorname{Herm}\left(\mathcal{R}^{n \times n}\right)$ 
and $\operatorname{Herm}\left(\mathcal{C}^{n \times n}\right)$, Theorem 1 reduces to the result of Crabtree and Haynsworth [4]. As mentioned in the Introduction, the proof given in [22], Theorem 1.4, continues to work for quaternions. Hence Theorem 1 is valid in $\operatorname{Herm}\left(\mathcal{H}^{n \times n}\right)$. Thus we need only to prove the result for matrices in $\operatorname{Herm}\left(\mathcal{O}^{3 \times 3}\right)$ with $r=3, k=2$, and $l=1$; this is what is presented below.

Proposition 1 Let $M \in \mathcal{J}=\operatorname{Herm}\left(\mathcal{O}^{3 \times 3}\right), C=E_{1}+E_{2}, D=E_{1}, U=P_{C}(M)$ be invertible in $\mathcal{J}(C, 1)$, and $A=P_{D}(U)$ be invertible in $\mathcal{J}(D, 1)$. Then $U / A=$ $P_{C-D}(M / A)$ is invertible in $\mathcal{J}(C-D, 1)$ and

$$
(M / A) /(U / A)=M / U
$$

Proof. In a nutshell, the result follows from the observations that $U / A$ and $(M / A) /(U / A)$ are scalar multiples of $E_{2}$ and $E_{3}$ respectively, and these scalars (which are determinants) are related, via (3), by

$$
\operatorname{det}((M / A) /(U / A))=\frac{\operatorname{det}(M / A)}{\operatorname{det}(U / A)}=\frac{\operatorname{det}(M) / \operatorname{det}(A)}{\operatorname{det}(U) / \operatorname{det}(A)}=\frac{\operatorname{det}(M)}{\operatorname{det}(U)}=\operatorname{det}(M / U) .
$$

To elaborate, consider the decompositions of the form (2) corresponding to the idempotents $C$ and $D$ :

$$
\begin{gathered}
M=U+V+W=\left[\begin{array}{lll}
p & a & 0 \\
\bar{a} & q & 0 \\
0 & 0 & 0
\end{array}\right]+\left[\begin{array}{lll}
0 & 0 & b \\
0 & 0 & c \\
\bar{b} & \bar{c} & 0
\end{array}\right]+\left[\begin{array}{lll}
0 & 0 & 0 \\
0 & 0 & 0 \\
0 & 0 & r
\end{array}\right], \\
U=A+B+C=\left[\begin{array}{lll}
p & 0 & 0 \\
0 & 0 & 0 \\
0 & 0 & 0
\end{array}\right]+\left[\begin{array}{lll}
0 & a & 0 \\
\bar{a} & 0 & 0 \\
0 & 0 & 0
\end{array}\right]+\left[\begin{array}{lll}
0 & 0 & 0 \\
0 & q & 0 \\
0 & 0 & 0
\end{array}\right],
\end{gathered}
$$

and

$$
M=A+Q+R=\left[\begin{array}{lll}
p & 0 & 0 \\
0 & 0 & 0 \\
0 & 0 & 0
\end{array}\right]+\left[\begin{array}{lll}
0 & a & b \\
\bar{a} & 0 & 0 \\
\bar{b} & 0 & 0
\end{array}\right]+\left[\begin{array}{lll}
0 & 0 & 0 \\
0 & q & c \\
0 & \bar{c} & r
\end{array}\right]
$$

(Here, $p, q$, and $r$ are real numbers, and $a, b$ and $c$ are octonions with overline denoting the conjugate.)

Now, for any two matrices $X$ and $Y$ in $\operatorname{Herm}\left(\mathcal{O}^{3 \times 3}\right)$,

$$
P_{X}(Y)=\frac{1}{2}\left[X(X Y)+X(Y X)+(X Y) X+(Y X) X-X^{2} Y-Y X^{2}\right] .
$$

Then a straightforward computation shows that

$$
M / U=W-P_{V}\left(U_{*}^{-1}\right)=\left[\begin{array}{lll}
0 & 0 & 0 \\
0 & 0 & 0 \\
0 & 0 & \lambda
\end{array}\right]
$$

where $\lambda=\frac{\operatorname{det}(M)}{\operatorname{det}(U)}$ (thanks to (3)). Similarly,

$$
M / A=R-P_{Q}\left(A_{*}^{-1}\right)=\left[\begin{array}{ccc}
0 & 0 & 0 \\
0 & q-p^{-1}|a|^{2} & c-p^{-1}(\bar{a} b) \\
0 & \bar{c}-p^{-1}(\bar{b} a) & r-p^{-1}|b|^{2}
\end{array}\right]
$$


and

$$
U / A=C-P_{B}\left(A_{*}^{-1}\right)=\left[\begin{array}{lcc}
0 & 0 & 0 \\
0 & q-p^{-1}|a|^{2} & 0 \\
0 & 0 & 0
\end{array}\right] .
$$

We note that $U / A$ is invertible in $\mathcal{J}(C-D, 1)=\mathcal{J}\left(E_{2}, 1\right)$ (which is the algebra generated by $E_{2}$ ) as its determinant (in this algebra) is $\operatorname{det}(U) / \operatorname{det}(A) \neq 0$. A straightforward computation shows that all entries of $(M / A) /(U / A)$ are zero except the $(3,3)$ entry which is

$$
\operatorname{det}((M / A) /(U / A))=\frac{\operatorname{det}(M / A)}{\operatorname{det}(U / A)}=\frac{\operatorname{det}(M) / \operatorname{det}(A)}{\operatorname{det}(U) / \operatorname{det}(A)}=\frac{\operatorname{det}(M)}{\operatorname{det}(U)}=\operatorname{det}(M / U) .
$$

It follows that $(M / A) /(U / A)=M / U$. This completes the proof of the proposition.

Remarks. In the above proof, we used (3) at various stages. Alternatively, one can use the Freudenthal determinant [17] given by

$$
\operatorname{det}(M)=p q r+2 \operatorname{Re}(\bar{b}(a c))-r|a|^{2}-q|b|^{2}-p|c|^{2} .
$$

In matrix theory, a real square matrix is said to be a $\mathbf{P}$-matrix if all its principal minors are positive. A result of Parsons [19] says that a (real square) matrix $M$ is a $\mathbf{P}$ matrix if and only if every principal pivotal transform of $M$ has positive diagonal. If $A$ is a nonsingular principal submatrix of $M$, then the principal pivotal transform of $M$ with respect to $A$ has two principal blocks $A^{-1}$ and $M / A$. Since a symmetric $\mathbf{P}$-matrix is positive definite, we may specialize Parsons' result as follows: A real symmetric matrix $M$ is positive definite if and only if for every principal submatrix $A$ of $M, A^{-1}$ and $M / A$ have positive diagonals. An analogue of this result is given below for Euclidean Jordan algebras. In what follows, when the Peirce decomposition $\sum_{1}^{r} x_{i} e_{i}+\sum_{i<j} x_{i j}$ of an $x$ with respect to the Jordan frame $\left\{e_{1}, e_{2}, \ldots, e_{r}\right\}$ is given, we say that $x_{i}$ s are the diagonal entries of $x$.

Corollary 1 Let $\mathcal{J}$ be simple and let $\left\{e_{1}, e_{2}, \ldots, e_{r}\right\}$ be a Jordan frame. For an $x \in \mathcal{J}$, consider the corresponding Peirce decomposition. Put $c_{k}:=e_{1}+e_{2}+\cdots+e_{k}$. The following conditions are necessary and sufficient for $x>0$ :

(i) $x$ has positive diagonal entries with respect to $\left\{e_{1}, e_{2}, \ldots, e_{r}\right\}$.

(ii) For all $k=1,2, \ldots, r, u^{(k)}:=P_{c_{k}}(x)$ is invertible in $\mathcal{J}\left(c_{k}, 1\right)$.

(iii) For all $k=1,2, \ldots, r-1, x / u^{(k)}>0$ has positive diagonal entries with respect to $\left\{e_{k+1}, e_{k+2}, \ldots, e_{r}\right\}$.

Proof. Clearly, conditions $(i)-($ iii $)$ are necessary for $x>0$. Now, suppose that these conditions are in place. By Theorem 1 , for each $k=2,3, \ldots, r, u^{(k)} / u^{(k-1)}=$ $P_{e_{k}}\left(x / u^{(k-1)}\right)$ is invertible in $\mathcal{J}\left(e_{k}, 1\right)$. Now, $u^{(k)} / u^{(k-1)}$ is of the form $\alpha_{k} e_{k}$ and so $\alpha_{k}$ is a diagonal element of $x / u^{(k-1)}$. By assumption, $\alpha_{k}>0$. Since

$$
\alpha_{k}=\operatorname{det}\left(u^{(k)} / u^{(k-1)}\right)=\frac{\operatorname{det}\left(u^{(k)}\right)}{\operatorname{det}\left(u^{(k-1)}\right)},
$$

we see that for all $k=1,2,3, \ldots, r$, $\operatorname{det}\left(u^{(k)}\right)$ have the same sign. Now, $u^{(1)}$ is of the form $\lambda e_{1}$, where $\lambda$ is a diagonal entry of $x$. By our assumption, $\lambda>0$, as $\lambda=\operatorname{det}\left(u^{(1)}\right)$, we see that the $\operatorname{determinants} \operatorname{det}\left(u^{(k)}\right)$ are all positive for $k=1,2, \ldots, r$. Since these are the "leading principal minors" of $x$, by Problem 5, VI.4 in [6] or Corollary 4.5 in [10], $x>0$. 


\section{Strict diagonal dominance of Schur complements}

In this section, we prove Theorem 2. We recall the Carlson-Markham theorem [1]: If $M$ is a complex strictly diagonally dominant matrix, then the Schur complement of any principal submatrix of $M$ in $M$ is also strictly diagonally dominant. In view of the Crabtree-Haynsworth quotient formula for matrices over quaternions (mentioned in the Introduction), the above result extends to matrices over quaternions with essentially the same proof.

We first prove the following

Lemma 2 Let the conditions of Theorem 2 be in place. Assume that $\mathcal{J}$ is simple and carries the trace inner product, and $k=1$. Then $x / u$ is strictly diagonally dominant with respect to $\left\{e_{2}, \ldots, e_{r}\right\}$.

Proof. In our setting,

$$
x=u+v+w=x_{1} e_{1}+\sum_{1<j \leq r} x_{1 j}+\sum_{2 \leq i \leq j \leq r} x_{i j} .
$$

The result is vacuously true for $r=1$. When $r=2$, we have $u=x_{1} e_{1}$ with $x_{1} \neq 0$ and $x / u=\lambda e_{2}$, where $\lambda=\operatorname{det}(x / u)=\frac{\operatorname{det}(x)}{\operatorname{det}(u)} \neq 0$ by (3). Hence, in this case, $x / u$ is strictly diagonally dominant. From now on, we assume that $r \geq 3$. We compute $x / u=w-P_{v}\left(x_{1}^{-1} e_{1}\right)$. Using the relations $x_{1 j} \circ e_{1}=\frac{1}{2} x_{1 j}$ and $x_{i j}^{2}=\frac{\left\|x_{i j}\right\|^{2}}{2}\left(e_{i}+e_{j}\right)$ for $i \neq j$ (see, [6], Prop. IV.1.4) we have

$$
2 v \circ\left(v \circ e_{1}\right)=v^{2}=\left[\sum_{k=2}^{r} \frac{\left\|x_{1 k}\right\|^{2}}{2}\right] e_{1}+\left[\sum_{k=2}^{r} \frac{\left\|x_{1 k}\right\|^{2}}{2} e_{k}\right]+2 \sum_{2 \leq i<j \leq r} x_{1 i} \circ x_{1 j} .
$$

Using the fact that $x_{1 i} \circ x_{1 j} \in \mathcal{J}_{i j}$ and the orthogonality of the Peirce spaces $\mathcal{J}_{i j}$ 's, we get

$$
P_{v}\left(e_{1}\right)=2 v \circ\left(v \circ e_{1}\right)-v^{2} \circ e_{1}=\left[\sum_{k=2}^{r} \frac{\left\|x_{1 k}\right\|^{2}}{2} e_{k}\right]+2 \sum_{2 \leq i<j \leq r} x_{1 i} \circ x_{1 j} .
$$

Thus,

$$
x / u=\sum_{k=2}^{r} z_{k} e_{k}+\sum_{2 \leq i<j \leq r} z_{i j}
$$

where

$$
z_{k}:=x_{k}-\frac{\left\|x_{1 k}\right\|^{2}}{2 x_{1}} \quad \text { and } \quad z_{i j}:=x_{i j}-\frac{2}{x_{1}} x_{1 i} \circ x_{1 j} .
$$

Therefore, proving the strict diagonal dominance of $x / u$ reduces to proving the inequalities

$$
\left|x_{k}-\frac{\left\|x_{1 k}\right\|^{2}}{2 x_{1}}\right|>\frac{1}{\sqrt{2}} \sum_{\Omega_{k}}\left\|x_{i j}-\frac{2}{x_{1}} x_{1 i} \circ x_{1 j}\right\|
$$

for all $k=2,3, \ldots, r$, where $\Omega_{k}:=\{(i, j): 2 \leq i<j \leq r, i=k$ or $j=k\}$. Now consider the real symmetric matrix $M=\left[m_{i j}\right]$ in $\mathcal{R}^{r \times r}$, where

$$
m_{i j}:=\left\{\begin{array}{c}
\sqrt{2}\left|x_{i}\right| \quad i=j \\
-\| x_{i j}|| i \neq j
\end{array}\right.
$$


By the strict diagonal dominance of $x$, we see that $M$ is a strictly diagonally dominant matrix. By the Carlson-Markham theorem (applied to real matrices), we see that the Schur complement $M /\left[\sqrt{2}\left|x_{1}\right|\right]$ is strictly diagonally dominant. Explicit computation of this matrix leads to the inequalities

$$
|\sqrt{2}| x_{k}\left|-\frac{\left\|x_{i k}\right\|^{2}}{\sqrt{2}\left|x_{1}\right|}\right|>\sum_{\Omega_{k}}\left[\left\|x_{i j}\right\|+\frac{1}{\sqrt{2} x_{1}}\left\|x_{1 i}\right\|\left\|x_{1 j}\right\|\right]
$$

for all $k=2,3, \ldots, r$. Now $\left\|x_{1 i} \circ x_{1 j}\right\|=\frac{1}{8}\left\|x_{1 i}\right\|\left\|x_{1 j}\right\|$ (which is valid in any simple algebra of rank greater than or equal to 3, see [6]), Lemma V.3.1). By using triangle and reverse triangle inequalities, (12) leads to (11). Thus we have proved the lemma.

$\square$

Proof of Theorem 2. In view of the reduction process, see Section 2, it is enough to prove the result for simple algebras. In a simple algebra, we can replace the given inner product by a positive multiple of the trace inner product. This will not result in any change in the Peirce decomposition of an object with respect to a Jordan frame. However, the strict diagonal dominance conditions simplify with $\left\|e_{k}\right\|=1$ for all $k$. From now on, we assume that $\mathcal{J}$ is simple and carries the trace inner product.

We are given that $x=u+v+w$ is strictly diagonally dominant with respect to $\left\{e_{1}, e_{2}, \ldots, e_{r}\right\}$, where $u=\sum_{1 \leq i \leq j \leq k} x_{i j}$, etc. Clearly, $u$ is strictly diagonally dominant with respect to $\left\{e_{1}, e_{2}, \ldots, e_{k}\right\}$. We now show that $x / u$ is strictly diagonally dominant with respect to $\left\{e_{k+1}, e_{k+2}, \ldots, e_{r}\right\}$. We prove the result by induction on $r$. In view of the above Lemma, we may assume that $1<k<r$. Let $a:=x_{1} e_{1}$. By applying the formula (4) with $d=e_{1}$ and $c=e_{1}+e_{2}+\cdots e_{k}$, we have $(x / a) /(u / a)=$ $x / u$. Now by the above Lemma, $x / a$ is strictly diagonally dominant with respect to $\left\{e_{2}, e_{3}, \ldots, e_{r}\right\}$ and $u / a$ is strictly diagonally dominant with respect to $\left\{e_{2}, e_{3}, \ldots, e_{k}\right\}$. By the induction hypothesis, $x / u=(x / a) /(u / a)$ is strictly diagonally dominant with respect to $\left\{e_{k+1}, e_{k+2}, \ldots, e_{r}\right\}$. This completes the proof.

\section{Schur product and a determinantal inequality}

Let $\mathcal{J}$ be any Euclidean Jordan algebra and $\left\{e_{1}, e_{2}, \ldots, e_{r}\right\}$ be a Jordan frame in $\mathcal{J}$. For any $x \in \mathcal{J}$, consider the Peirce decomposition of $x$ with respect to this Jordan frame:

$$
x=\sum_{i \leq j} x_{i j} .
$$

We note that $x_{i i}=x_{i} e_{i}$ and $x_{i j}=x_{j i} \in \mathcal{J}_{i j}$ for all indices $i, j \in\{1,2, \ldots, r\}$. For any $A=\left[a_{i j}\right] \in \operatorname{Herm}\left(\mathcal{R}^{r \times r}\right)$, we define the Schur product of $A$ and $x$ by:

$$
A \bullet x:=\sum_{i \leq j} a_{i j} x_{i j}
$$

This induces a transformation on $\mathcal{J}$ :

$$
W_{A}(x)=A \bullet x .
$$

Given $A, B \in \operatorname{Herm}\left(\mathcal{R}^{r \times r}\right)$ and real $\lambda$, the following are obvious:

(1) The mapping $(A, x) \rightarrow A \bullet x$ is bilinear, 
(ii) $W_{A}: \mathcal{J} \rightarrow \mathcal{J}$ is linear, and

(iii) $W_{A+B}=W_{A}+W_{B}$ and $W_{\lambda A}=\lambda W_{A}$.

Here are some examples.

Example 1. Let $\mathcal{J}=\operatorname{Herm}\left(\mathcal{R}^{n \times n}\right)$ and consider the canonical Jordan frame $\left\{E_{1}, E_{2}, \ldots, E_{n}\right\}$. With respect to this Jordan frame, any matrix in $\mathcal{J}$ will have its usual matrix representation. Then for $A, X \in \operatorname{Herm}\left(\mathcal{R}^{n \times n}\right), A \bullet X$ is the usual Schur product of two real symmetric matrices.

Example 2. Given $r$ real numbers $a_{1}, a_{2}, \ldots, a_{r}$, let $A=\left[a_{i j}\right]$ with $a_{i j}:=\frac{a_{i}+a_{j}}{2}$ for all $i, j$. Then

$$
A \bullet x=\sum_{i \leq j} \frac{a_{i}+a_{j}}{2} x_{i j}=L_{a}(x):=a \circ x,
$$

where $a=\sum_{1}^{r} a_{i} e_{i}$ and $L_{a}$ is the Lyapunov transformation corresponding to $a$.

Example 3. Given $r$ real numbers $a_{1}, a_{2}, \ldots, a_{r}$, let $A=\left[a_{i j}\right]$ with $a_{i j}:=a_{i} a_{j}$ for all $i, j$. Then

$$
A \bullet x=\sum_{i \leq j} a_{i} a_{j} x_{i j}=P_{a}(x),
$$

where $a=\sum_{1}^{r} a_{i} e_{i}$ and $P_{a}$ is the quadratic representation corresponding to $a$.

Example 4. Given a real valued differentiable function $\phi: \mathcal{R} \rightarrow \mathcal{R}$, consider the corresponding Löwner function $\Phi: \mathcal{J} \rightarrow \mathcal{J}$ defined by (the spectral decompositions)

$$
a=\lambda_{1} e_{1}+\lambda_{2} e_{2}+\cdots+\lambda_{r} e_{r} \quad \text { and } \quad \Phi(a)=\phi\left(\lambda_{1}\right) e_{1}+\phi\left(\lambda_{2}\right) e_{2}+\cdots+\phi\left(\lambda_{r}\right) e_{r} .
$$

Then the directional derivative of $\Phi$ at $a=\sum \lambda_{i} e_{i}$ in the direction of $x=\sum x_{i j}$ (Peirce decomposition written with respect to $\left\{e_{1}, e_{2}, \ldots, e_{r}\right\}$ ) is given by

$$
\Phi^{\prime}(a ; x):=\sum_{i \leq j} a_{i j} x_{i j}
$$

where $a_{i j}:=\frac{\phi\left(\lambda_{i}\right)-\phi\left(\lambda_{j}\right)}{\lambda_{i}-\lambda_{j}}$ (which, by convention, is the derivative of $\phi$ when $\lambda_{i}=\lambda_{j}$ ). We now note that $\Phi^{\prime}(a ; x)=A \bullet x$, where $A=\left[a_{i j}\right]$.

Theorem 3 If $A$ is a nonnegative matrix, then $W_{A}$ is monotone. The converse holds if $\mathcal{J}$ is simple.

Proof. If $A$ is a nonnegative matrix, the expression

$$
\left\langle W_{A}(x), x\right\rangle=\sum_{i \leq j} a_{i j}\left\|x_{i j}\right\|^{2}
$$

is nonnegative for all $x$. This shows that $W_{A}$ is monotone. Now assume that $W_{A}$ is monotone. Then, because $\mathcal{J}$ is simple, for any $i \leq j$, Peirce space $\mathcal{J}_{i j}$ is nonzero (see [6], Prop. IV.2.3). Taking a nonzero $x_{i j} \in \mathcal{J}_{i j}$, the inequality $0 \leq\left\langle W_{A}\left(x_{i j}\right), x_{i j}\right\rangle=$ $a_{i j}\left\|x_{i j}\right\|^{2}$ shows that $a_{i j} \geq 0$. This implies that all entries of $A$ are nonnegative.

The following result generalizes Schur's classical theorem on Schur products [13] to the setting of Euclidean Jordan algebras. Its proof is essentially given in [14]. In what follows, we use the notation $A \succeq 0$ to mean that $A$ is Hermitian and positive semidefinite. 
Theorem 4 Suppose that $A \succeq 0$ in $\operatorname{Herm}\left(\mathcal{R}^{r \times r}\right)$ and $x \geq 0$ in $\mathcal{J}$. Then $A \bullet x \geq 0$ in $\mathcal{J}$.

Proof. As $A$ is symmetric and positive semidefinite, it can be written as a nonnegative linear combination of finite number of matrices of the form $s s^{T}$, where $s$ is a (column) vector in $\mathcal{R}^{r}$. Our result follows once we show that $s s^{T} \bullet x \geq 0$ in $\mathcal{J}$. Now, if $s_{i}$ $(i=1,2, \ldots, r)$ are the components of $s$, then

$$
s s^{T} \bullet x=\sum_{i \leq j} s_{i} s_{j} x_{i j}=P_{a}(x),
$$

where $a:=\sum_{1}^{r} s_{i} e_{i}$. Since $P_{a}$ (more generally, any quadratic representation) keeps the symmetric cone $K$ invariant and $x \geq 0$, we see that $P_{a}(x) \geq 0$. This completes the proof.

In the context of real symmetric positive semidefinite matrix $A=\left[a_{i j}\right]$, the wellknown Hadamard inequality states that

$$
\operatorname{det}(A) \leq a_{11} a_{22} \cdots a_{r r}
$$

An analogous result holds in any Euclidean Jordan algebra [10]: If $x \geq 0$ in $\mathcal{J}$ with Peirce decomposition $x=\sum_{i \leq j} x_{i j}$ with respect to any Jordan frame $\left\{e_{1}, e_{2}, \ldots, e_{r}\right\}$, then

$$
\operatorname{det}(x) \leq x_{1} x_{2} \cdots x_{r}
$$

where $x_{i i}=x_{i} e_{i}$, etc. The following inequality, which generalizes the so-called Oppenheim inequality of matrix theory (see [21], Theorem 6.25), gives a lower bound on the determinant of $A \bullet x$.

Theorem 5 Suppose $A=\left[a_{i j}\right] \succeq 0$ in $\operatorname{Herm}\left(\mathcal{R}^{r \times r}\right)$ and $x \geq 0$ in $\mathcal{J}$. Then

$$
\left(a_{11} a_{22} \cdots a_{r r}\right) \operatorname{det}(x) \leq \operatorname{det}(A \bullet x) \leq\left(a_{11} a_{22} \cdots a_{r r}\right)\left(x_{1} x_{2} \cdots x_{r}\right) .
$$

Proof. The inequality on the right comes from the generalized Hadamard inequality (13) applied to $A \bullet x$; we prove only the inequality on the left. Also, by a continuity argument, it is enough to prove the result when $A$ is positive definite and $x>0$ in $\mathcal{J}$. Our proof is by induction on the rank $r$ of $\mathcal{J}$. There is nothing to prove when $r=1$, so assume $r>1$. Let $c=e_{1}$, where $\left\{e_{1}, e_{2}, \ldots, e_{r}\right\}$ is the given Jordan frame with respect to which $A \bullet x$ is defined. Corresponding to this, consider the Peirce decomposition (2):

$$
x=u+v+w,
$$

where $u=x_{1} e_{1}$; Analogously, we write the Peirce decomposition of $A$ with respect to the idempotent $E_{1}$ in $\operatorname{Herm}\left(\mathcal{R}^{r \times r}\right)$ :

$$
A=A_{11}+A_{12}+A_{22}
$$

where $A_{11}=a_{11} E_{1}$. Now consider

$$
y:=u+v+P_{v}\left(u_{*}^{-1}\right) .
$$

Since $u>0$ in $V(c, 1)$ and $y / u=P_{v}\left(u_{*}^{-1}\right)-P_{v}\left(u_{*}^{-1}\right)=0$, by Item (ii) of the Introduction, $y \geq 0$. By the previous theorem, $A \bullet y \geq 0$ in $\mathcal{J}$. We write the Peirce decomposition

$$
A \bullet y=p+q+r
$$


where $p=A_{11} \bullet u, q=A_{12} \bullet v$, and $r=A_{22} \bullet P_{v}\left(u_{*}^{-1}\right)$. As $p=a_{11} x_{1} e_{1}$ is invertible in $V(c, 1)$ by Item $(i i)$ of the Introduction,

$$
r-P_{q}\left(p_{*}^{-1}\right)=(A \bullet y) / p \geq 0 .
$$

This implies, as $r=A_{22} \bullet P_{v}\left(u_{*}^{-1}\right)=A_{22} \bullet(w-x / u)$,

$$
A_{22} \bullet w-P_{q}\left(p_{*}^{-1}\right) \geq A_{22} \bullet x / u \text {. }
$$

Now,

$$
0<A \bullet x=A_{11} \bullet u+A_{12} \bullet v+A_{22} \bullet w=p+q+A_{22} \bullet w
$$

implies $(A \bullet x) / p=A_{22} \bullet w-P_{q}\left(p_{*}^{-1}\right)$. Thus (14) becomes

$$
(A \bullet x) / p \geq A_{22} \bullet(x / u) .
$$

Now using the well-known inequality in Euclidean Jordan algebra theory, namely, $u \geq$ $v \geq 0 \Rightarrow \operatorname{det}(u) \geq \operatorname{det}(v)$ (see [6], Exercise 4, Page 59), we get

$$
\operatorname{det}((A \bullet x) / p) \geq \operatorname{det}\left(A_{22} \bullet(x / u)\right) .
$$

By the induction hypothesis, $\operatorname{det}\left(A_{22} \bullet(x / u)\right) \geq\left(a_{22} a_{33} \cdots a_{r r}\right) \operatorname{det}(x / u)$. By the determinantal formula (3) applied to $(A \bullet x) / p$ and $x / u$, and the observation $\operatorname{det}(p)=$ $\operatorname{det}\left(a_{11} x_{1} e_{1}\right)=a_{11} x_{1}$, we have

$$
\operatorname{det}(A \bullet x) \geq a_{11} x_{1}\left(a_{22} a_{33} \cdots a_{r r}\right) \operatorname{det}(x) / \operatorname{det}(u) .
$$

As $\operatorname{det}(u)=x_{1}$, this simplifies to

$$
\operatorname{det}(A \bullet x) \geq a_{11} a_{22} a_{33} \cdots a_{r r} \operatorname{det}(x) .
$$

This completes the proof.

The following result is immediate in view of the Hadamard inequality.

Corollary 2 If $A \succeq 0$ and $x \geq 0$, then $\operatorname{det}(A) \operatorname{det}(x) \leq \operatorname{det}(A \bullet x)$.

Our next result is an interesting consequence of Theorem 4, although it is not related to Schur complements. In this result, for any given set of numbers $\left\{b_{1}, b_{2}, \ldots, b_{r}\right\}$, we denote the decreasing rearrangement by $\left\{b_{1}^{\downarrow}, b_{2}^{\downarrow}, \ldots, b_{r}^{\downarrow}\right\}$; in particular, for $z \in \mathcal{J}$, $\lambda_{i}^{\downarrow}(z)(i=1,2, \ldots, r)$ denote the eigenvalues of $z$ written in the decreasing order. We use the familiar notation $\lambda_{1}^{\downarrow}(z)=\lambda_{\max }(z)$ and $\lambda_{r}^{\downarrow}(z)=\lambda_{\min }(z)$.

Theorem 6 Let $\mathcal{J}$ be simple. Let $A=\left[a_{i j}\right] \succeq 0$ in $\operatorname{Herm}\left(\mathcal{R}^{r \times r}\right)$ and $x \geq 0$ in $\mathcal{J}$. Then for all $i=1,2, \ldots, r$,

$$
a_{i i}^{\downarrow} \lambda_{\min }(x) \leq \lambda_{i}^{\downarrow}(A \bullet x) \leq a_{i i}^{\downarrow} \lambda_{\max }(x) .
$$

Proof. For notational simplicity, let $\alpha:=\lambda_{\min }(x)$ and $\beta:=\lambda_{\max }(x)$. Then $x-\alpha e \geq 0$ and $\beta e-x \geq 0$ in $\mathcal{J}$. By Theorem $4, A \bullet(x-\alpha e) \geq 0$ and $A \bullet(\beta e-x) \geq 0$. These two yield

$$
\alpha A \bullet e \leq A \bullet x \leq \beta A \bullet e .
$$

Now, $e=\sum_{1}^{r} e_{i}$ (where $\left\{e_{1}, e_{2}, \ldots, e_{r}\right\}$ is the Jordan frame with respect to which $A \bullet x$ is defined) and so $\alpha(A \bullet e)=\sum_{1}^{r} \alpha a_{i i} e_{i}$; a similar expression holds for $\beta(A \bullet e)$. Since $\beta \geq \alpha \geq 0$, using the well-known fact that $y \leq z$ in $\mathcal{J}$ implies $\lambda_{i}^{\downarrow}(y) \leq \lambda_{i}^{\downarrow}(z)$ for all $i$ (which follows immediately from Hirzebruch's theorem, see [12]), we have

$$
\alpha a_{i i}^{\downarrow} \leq \lambda_{i}^{\downarrow}(A \bullet x) \leq \beta a_{i i}^{\downarrow} .
$$

This completes the proof. 


\section{References}

1. D. Carlson and T.L. Markham, Schur complements of diagonally dominant matrices, Czech. Math. Jour., 29 (1979) 246-251.

2. N. Cohen and S. De Leo, The quaternionic determinant, Electron. Jour. Lin. Alg., 7 (2000) $100-111$.

3. R.W. Cottle, On manifestations of the Schur complement, Rend. Sem. Mat. Fis. Milano, 45 (1975) 31-40.

4. D.E. Crabtree and E.V. Haynsworth, An identity for the Schur complement of a matrix, Proc. of Amer. Math. Soc., 22 (1969) 364-366.

5. T. Dray and C.A. Manogue, The octonionic eigenvalue problem, Adv. Appl. Clifford Alg., 8 (1998) 341-364.

6. J. Faraut and A. Korányi, Analysis on Symmetric Cones, Clarendon Press, Oxford, 1994

7. M.S. Gowda and R. Sznajder, Automorphism invariance of $\mathbf{P}$ and GUS properties of linear transformations on Euclidean Jordan algebras, Math. Oper. Res., 31 (2006) 109123.

8. M.S. Gowda and R. Sznajder, Schur complements, Schur determinantal and Haynsworth inertia formulas in Euclidean Jordan algebras, Linear Alg. Appl., 432 (2010) 1553-1559.

9. M.S. Gowda, R. Sznajder, and J. Tao, Some P-properties for linear transformations on Euclidean Jordan algebras, Linear Alg. Appl., 393 (2004) 203-232.

10. M.S. Gowda and J. Tao, The Cauchy interlacing theorem in simple Euclidean Jordan algebras and some consequences, Linear and Multilinear Alg., to appear.

11. M.S. Gowda and J. Tao, Some inequalities in Euclidean Jordan algebras, Manuscript, December 2009

12. M.S. Gowda, J. Tao, and M. Moldovan, Some inertia theorems in Euclidean Jordan algebras, Linear Alg. Appl., 430 (2009) 1992-2011.

13. R.A. Horn and C.R. Johnson, Matrix Analysis, Cambridge University Press, Cambridge, 1985.

14. A. Korányi, Monotone functions on formally real Jordan algebras, Math. Ann., 269 (1984) 73-76.

15. O. Loos, Finiteness condition in Jordan pairs, Math. Z., 206 (1991) 577-587.

16. H. Massam and E. Neher, On transformations and determinants of Wishart variables on symmetric cones, Jour. Theoret. Probab., 10 (1997) 867-902.

17. M.M. Moldovan and M.S. Gowda, Strict diagonal dominance and a Gers̆gorin type theorem in Euclidean Jordan algebras, Linear Alg. Appl., 431 (2009) 148-161.

18. D.V. Ouellette, Schur complements and statistics, Linear Alg. Appl., 36 (1981) 187-295.

19. T.D. Parsons, Applications of principal pivoting, Proc. of Princeton Symposium on Mathematical Programming, Princeton University Press, Princeton, New Jersey, 567-581, 1970.

20. S.H. Schmieta and F. Alizadeh, Extension of primal-dual interior point algorithms to symmetric cones, Math. Prog., Series A, 96 (2003) 409-438.

21. F. Zhang, Matrix Theory, Springer, New York, 1999.

22. F. Zhang, The Schur Complement and its Applications, Springer, New York, 2005. 